\title{
VORWORT DER HERAUSGEBER DIESES BANDES
}

Die in diesem Band enthaltenen Referate und Diskussionsbeiträge wurden auf einer ersten von mehreren geplanten Tagungen über die Philosophie Friedrich Nietzsches vorgetragen. Als Thema dieser Tagung war Nietzsches Auseinandersetzung mit wesentlichen Tendenzen seiner Zeit vorgesehen. Im Zuge der Vorbereitung erwies es sich sehr rasch als unmöglich, im Rahmen von drei Tagen diesem Thema in zureichendem Maße gerecht zu werden. Nach den ersten Korrespondenzen und nach Sichtung der Vorschläge von eingeladenen Referenten zogen wir es vor, einige ausgewählte Aspekte etwas ausführlicher zu behandeln. Dies gilt in besonderem Maße für das Thema Nietzsche und die Romantik. Als weitere Schwerpunkte kristallisierten sich in den Vorverhandlungen heraus: Nietzsches Verhältnis zur Psychologie, zu den Naturwissenschaften und zur Musik. Selbst hinsichtlich dieser Schwerpunkte konnten wir keine Vollständigkeit anstreben; andere für Nietzsches Beziehung zu seinem Jahrhundert wichtige Themen mußten völlig ausgeklammert werden. Obwohl viele Fragen, die Nietzsches Stellung zu seiner Zeit betreffen, unerörtert bleiben mußten, wird das Thema einer zweiten Tagung, die für den Sommer 1980 vorgesehen ist, wahrscheinlich die Wirkungsgeschichte von Nietzsches Denken in unserem Jabrhundert bilden.

Im Verlauf der Tagung, besonders deutlich in der Schlußaussprache, wurde von mehreren Teilnehmern moniert, daß wir, trotz der in der Vorbereitungsphase eingetretenen Veränderungen, den ursprünglichen $\mathrm{Ar}$ beitstitel Nietzsche als Kritiker seines Jabrhunderts für die Tagung beibehalten haben. Wir tragen dieser berechtigten Kritik nunmehr Rechnung, indem wir diesen Band unter einem anderen Titel veröffentlichen. Mit der Formulierung Aneignung und Umwandlung wollen wir einen Grundzug von Nietzsches Umgang mit der Tradition herausheben, auf den von vielen Teilnehmern mehrfach eingegangen worden ist: daß Nietzsche nie bloß rezipiert, vielmehr das Aufgenommene von der ersten Lektüre oder Kenntnisnahme an in ein unverwechselbar Eigenes umgeprägt hat. Das trifft auf das von ihm Begrüßte wie auf das von ihm Verworfene zu.

Auf S. XV des Bandes werden die Teilnehmer der Tagung aufgeführt. Nach der Festlegung von Themen und Referenten zu einem ziemlich frühen Zeitpunkt beachteten wir bei den zusätzlichen Einladungen vor allem zwei Gesichtspunkte. Zum einen sollten neben der Philosophie auch andere an 
Nietzsches Verhältnis zu seinem Jahrhundert besonders interessierte Disziplinen, z. B. Germanistik, Literaturwissenschaft, Theologie und Musikwissenschaft durch Sachkenner vertreten sein. Zum anderen versuchten wir, die Einladungen im Blick auf die Besonderheit der Schwerpunkte auszusprechen. Von den vorgesehenen Teilnehmern, die uns zugesagt hatten, waren schließlich drei zu unserem Bedauern verhindert. Prof. Dr. Reiner Wiehl (Philosophie, Heidelberg) und Prof. Dr. Peter Köster (Theologie, Bielefeld) erkrankten in letzter Minute; Prof. Dr. Ferruccio Masini (Germanistik, Rom) erhielt durch ein Mißverständnis die Einladung mit den genauen Terminen zu spät. - Von den auf der Liste Aufgeführten haben nicht alle an der gesamten Tagung teilnehmen können. Einige reisten mit Verspätung an, am zweiten oder gar erst am dritten Tag der Tagung; andere mußten Berlin vorzeitig verlassen.

Die Referate sind in einer Reihe von Fällen umfassender und detaillierter ausgearbeitet worden, als sie - aus Zeitgründen - während der Tagung vorgetragen wurden. Der Abdruck in diesem Band erfolgt den jeweiligen Wünschen der Autoren gemäß. Selbstverständlich sind in der Druckfassung auch Anmerkungen und Stellenverweise mit aufgenommen worden. Auch sind einige Referate nach der Tagung noch überarbeitet und ergänzt worden; solche Ergänzungen und Uberarbeitungen wurden nur berücksichtigt, wenn sie sich nicht aus den Diskussionen ergaben oder nahelegten. - Von einer Vereinheitlichung der Vorträge hinsichtlich der benutzten Ausgaben und der Zitierweise haben wir, dem generellen Brauch der Nietzsche-Studien folgend, abgesehen.

Die Diskussionen wurden während der Tagung aufgezeichnet. Die Abschriften der Tonbandmitschnitte haben wir überarbeitet, d. h. gestrafft und in einigen Fällen in eine andere Abfolge gebracht, die sich aus Sachgründen anbot. Wir legten aber Wert darauf, die Argumente der Diskussionsteilnehmer nicht zu verändern und ihre sprachlichen Eigentümlichkeiten nach Möglichkeit zu bewahren. Nach unserer Úberarbeitung hat jeder Diskutant seine Voten noch einmal überprüft. Fast alle haben von der Möglichkeit Gebrauch gemacht, einzelne Sätze oder Passagen zu präzisieren bzw. sie stärker ihrem persönlichen Stil anzugleichen. - Einige Diskussionen mußten während der Tagung aus Zeitmangel begrenzt oder abgebrochen werden. Das hat dazu geführt, daß manches allgemeine Problem nicht in zureichender Ausführlichkeit besprochen werden konnte. Beispiele dafür sind die mehrfach aufflackernden Gespräche über die Bedeutung und das Recht der Einbeziehung des Nachlasses Nietzsches, sowie über die Frage, ob sich in Nietzsches Werk faschistische Ideen oder Tendenzen aufweisen lassen. Auf den Abdruck dieser Diskussionsansätze wollten wir gleichwohl nicht verzichten. - Auf Belege und Zitatnachweise für Diskussionsvoten haben wir, 
von wenigen Ausnahmen abgesehen, verzichtet, um deren Lebendigkeit und Freiheit auch für den Leser ausdrücklich werden zu lassen.

Unser Dank gilt allen denen, die zum Zustandekommen und Gelingen der Tagung und dieses Bandes beigetragen haben. Das ist in erster Linie die Fritz Thyssen Stiftung. Von ihrem Repräsentanten, Herrn Prof. Dr. Gerd Brand, ging die Anregung zu den Nietzsche-Tagungen aus. Die Stiftung hat durch ihre finanzielle Unterstützung die Durchführung zunächst dieser ersten Tagung möglich gemạcht. Unser Dank gilt allen Teilnehmern an der Tagung, insbesondere denen, die durch Referate und Diskussionsbeiträge zu ihrer Gestaltung beigetragen haben. Später haben sie durch rasche und termingerechte Durchsicht und Uberarbeitung der Referate und Diskussionsvoten mitgeholfen, daß dieser Band dem Publikum nicht allzu lange nach Beendigung der Tagung vorgelegt werden kann. Besonderer Dank geht an Herrn Janz, der es ermöglichte, Tonbandaufzeichnungen von zwei Musikstücken Nietzsches - Ermanarich und Der Hymnus an die Freundschaft anzuhören; im Zusammenhang mit seinem Vortrag vermittelten sie einen instruktiven Eindruck von Nietzsches kompositorischen Arbeiten.

Wir danken ferner unseren Mitarbeitern Norbert Gebert, Marie-Luise Haase und Johannes Neininger, die zu einem reibungslosen Ablauf des Kongresses und zur technischen Vorbereitung dieses Bandes viel beigetragen haben.

Im Januar 1978

Müller-Lauter Salaquarda 
